\title{
Efeito Antioxidante da Ilex paraguariensis e Condimentos na Oxidação Lipídica de Filés de Oreochromis niloticus
}

\author{
Amanda A. R. Machado ${ }^{1}$, Nilce Coelho Peixoto ${ }^{2}$, Tiffany P. Hautrive ${ }^{1}$, \\ Juliano Uczay ${ }^{1}$, Aline S. Bezerra ${ }^{1}$
}

\begin{abstract}
RESUMO
Objetivo: os compostos antioxidantes presentes naturalmente nas plantas têm sido alvo de pesquisas em substituição aos sintéticos como aditivos alimentícios. Este estudo teve por objetivo avaliar o potencial antioxidante de extrato de erva-mate, em associação com os condimentos alecrim, orégano e tomilho, em filés de tilápia submetidos a diferentes formas de preparo, ante a oxidação lipídica do pescado. Método: os extratos foram avaliados quanto ao percentual de redução do radical 2,2-difenil-1-picril-hidrazil e quanto aos teores de compostos fenólicos totais. A oxidação lipídica do pescado foi analisada pelo teste das substâncias reativas ao ácido tiobarbitúrico (TBARS, do inglês, thiobarbituric acid reactive substances). Resultados: os resultados mostraram que o orégano foi o condimento que apresentou o maior teor de compostos fenólicos totais, e a erva-mate a maior atividade antioxidante. Com relação à análise de TBARS, na adição do mix de erva-mate com os três condimentos aos filés de tilápia in natura e submetidos aos diferentes processamentos térmicos (frito ou assado), foi verificado que apresentaram os menores valores relativos às amostras controle (sem a adição do mix), atingindo uma diminuição de, aproximadamente, dez vezes no pescado preparado assado. Quanto ao reaquecimento, foi constatado que este fator dobra a quantidade de TBARS presentes. Conclusões: assim, conforme os resultados, é possível sugerir a eficácia da associação dos condimentos com a erva-mate na redução da rancidez oxidativa dos filés de tilápia submetidos aos diferentes processamentos térmicos aplicados, bem como do produto in natura.

Palavras-chave: Ilex paraguariensis. Especiarias. Perciformes.

ANTIOXIDANT EFFECT OF ILEX PARAGUARIENSIS AND CONDIMENTS ON LIPID OXIDATION OF OREOCHROMIS NILOTICUS STEAK ABSTRACT

Objective: the antioxidant compounds naturally present in plants have been the subject of research replacing synthetic ones as food additives. The objective of this study was to evaluate the antioxidant potential of yerba mate extract, in association with rosemary, oregano and thyme condiments, in tilapia fillets submitted to different preparation forms, in face of lipid oxidation of fish. Method: the extracts were evaluated for the percentage reduction of the 2,2-diphenyl-1-picryl-hydrazyl radical and for the content of total phenolic compounds. The lipid oxidation of the fish was analyzed by thiobarbituric acid reactive substances (TBARS) test. Results: the results showed that the oregano was the condiment that presented the highest content of total phenolic compounds and the yerba mate, the highest antioxidant activity. Regarding the analysis of TBARS, the addition of the yerba mate mix with the three condiments to the fillets of in natura tilapia and submitted to the different thermal processes (fried or roasted), were verified that presented the smaller values relative to the control samples (without the addition of the mix), reaching a reduction of approximately ten times in prepared roasted fish. As for reheating, it was found that this factor doubles the amount of TBARS present. Conclusions: thus, according to the results, it is possible to suggest the effectiveness of the association of condiments with yerba mate in the reduction of oxidative rancidity of the tilapia fillets submitted to the different thermal processes applied, as well as the in natura product.
\end{abstract}

Keywords: Ilex paraguariensis. Spices. Perciformes.

RECEBIDO EM: 8/6/2019

MODIFICAČ̃̃ES SOLICITADAS EM: 10/9/2019

ACEITO EM: 26/2/2020

\footnotetext{
Universidade Federal de Santa Maria, Campus Palmeira das Missões. Palmeira das Missões/RS, Brasil.

Autora correspondente. Universidade Federal de Santa Maria - UFSM. Campus Palmeira das Missões. Av. Independência, 3751, Bairro Vista Alegre, Palmeira das Missões/RS, Brasil. CEP: 98300-000. http://lattes.cnpq.br/6573904661865745. https://orcid.org/0000-0002-6982-867X. ncpeixoto@yahoo.com.br
} 


\section{INTRODUÇÃO}

A tilápia do Nilo (Oreochromis niloticus Linnaeus) é um pescado de grande importância mundial no setor de aquicultura. É considerada uma das espécies mais indicadas para a criação intensiva, por possuir rápido crescimento, maturação sexual tardia, hábito alimentar onívoro e ser de grande rusticidade. Mercadologicamente, além de ser um peixe de baixo valor comercial e de filetagem descomplicada, sua comercialização é facilitada em virtude de sua carne branca, textura firme, sabor delicado e ausência de espinhas intramusculares, o que gera uma considerável aceitabilidade pelo público consumidor (WANG; LU, 2016). No Brasil, esta é a espécie mais cultivada na aquicultura interior, com uma produção anual superior a 283 mil toneladas (IBGE, 2017).

O alto teor lipídico da carne da tilápia, contudo, é um fator desfavorável e deve ser considerado, pois leva à rápida deterioração sensorial e nutricional se não for manuseada, transportada e armazenada de maneira correta. A rancidez oxidativa é consequência da metabolização dos lipídios pelos microrganismos que se proliferam no produto (EVANGELISTA-BARRETO et al., 2017).

Conforme Kanner (2007), o nosso trato gastrointestinal é exposto a compostos químicos oxidados pela sua ingestão por meio da dieta. 0 consumo de gorduras oxidadas por animais e humanos acarreta em aumento na excreção urinária de malondialdeído (MDA) e de compostos carbonilados, marcadores de danos oxidativos (lipídico e proteico, respectivamente). Além destes exemplificados, nosso metabolismo normal, por intermédio de rotas bioquímicas, desencadeia a formação de quantidades pequenas destes compostos nocivos (MURUSSI et al., 2014; KANNER, 2007).

Dados mostram que os polifenóis ingeridos com alimentos contendo moléculas lipídicas susceptíveis à oxidação, impedem sua conversão metabólica em produtos perniciosos (VISIOLI, 2014). Ainda, o MDA e outros produtos da oxidação têm despertado a atenção da comunidade científica por sugerir sua relação com o desenvolvimento de diversos tipos de neoplasias. Conforme estudo realizado por Kangari et al. (2018), o MDA é indicado como um dos mais abundantes e importantes aldeídos da peroxidação lipídica, podendo reagir com proteínas, DNA e outras biomoléculas e, assim, alterar sua estrutura e função, evoluindo para um tumor. Mediante avaliação dos níveis de produtos oxidados nos tecidos e no plasma, foi verificado que estes são significativamente maiores em pacientes com câncer e em indivíduos tabagistas comparativamente com pacientes saudáveis, levando à dedução de que o estresse oxidativo, induzido por um desequilíbrio nas defesas antioxidantes e/ou a produção exacerbada de tais moléculas/radicais, podem ser um fator predisponente para tal patologia (HORN et al., 2015; KANGARI et al., 2018).

A erva-mate (EM (Ilex paraguariensis St. Hil.)), originária da América do Sul, é usada no chimarrão, bebida tradicionalmente presente no cotidiano dos gaúchos, na região dos pampas, incluindo o Rio Grande do Sul. Por seu alto consumo, e aliado aos possíveis benefícios advindos deste, diversos estudos analisam o potencial antioxidante do seu extrato e a possível aplicação em alimentos (BATTISTON et al., 2016; COLPO et al., 2016).

A EM contém compostos químicos que apresentam propriedades antioxidantes. Entre os exemplares estão os compostos aromáticos bioativos, cuja aplicação pode controlar a oxidação lipídica durante as fases de processamento e armazenamento dos produtos, beneficiando a indústria de alimentos (BATTISTON et al., 2016; COLPO et al., 2016).

No mesmo sentido, plantas condimentares, como salsa, manjericão, alho, cebola, coentro e orégano, possuem propriedades antioxidantes atribuídas, entre outros fatores, à presença de compostos fenólicos e flavonoides e, além disso, sua ingestão é adequada à população hipertensa, pela substituição das especiarias e por dispensar ou diminuir o emprego de sal na dieta, o que teve boa aceitação pelos sujeitos pesquisados (MENDES; RODRIGUES-DAS-DORES; CAMPIDELI, 2015). Plantas alimentícias, como almeirão (Cichorium intybus) e radite (ou radicchio, radiche, Hypochaeris chillensis) também têm um conteúdo considerável de compostos fenólicos e boa capacidade antioxidante, como já investigado por este grupo de pesquisa (BEZERRA et al., 2017).

Os vegetais (incluindo especiarias, condimentos, ervas e alimentos) que contêm quantidades significativas de substâncias fenólicas e, consequentemente, dispõem do atributo antioxidante, são promissores como alimentos funcionais e por isso os aditivos sintéticos são preteridos. O mecanismo pelo qual a propriedade é atribuída conta com a capacidade de quelar espécies reativas e radicais livres endógenos ou exógenos, o que possibilita sua neutralização, conferindo um adicional ao sistema de defesa e impedimento de suas consequências danosas à saúde de quem os ingere (BLUM-SILVA et al., 2015; COLPO et al., 2016; ZAWADZKI et al., 2017; WEBER, 2018). 
Deste modo, o objetivo do presente estudo foi avaliar o potencial antioxidante in vitro de extratos de EM em associação com condimentos reconhecidamente eficazes como antioxidantes e que melhoram o aroma e o sabor em filés de tilápia submetidos a diferentes modos de preparo.

\section{MÉTODO}

Trata-se de um estudo experimental, executado na Universidade Federal de Santa Maria, Campus de Palmeira das Missões, RS. A elaboração dos extratos foi realizada no Laboratório de Apoio à Nutrição, e o processamento dos filés de pescado no Laboratório de Técnica Dietética. As análises químicas, feitas em triplicata, foram conduzidas no Laboratório de Bromatologia.

A amostra de EM (I. paraguariensis) foi doada por uma indústria ervateira do município de Venâncio Aires, situado no Estado do Rio Grande do Sul. A amostra era $100 \%$ nativa e isenta de açúcar e glúten.

Os condimentos e especiarias (alecrim, orégano e tomilho), pulverizados e obtidos por meio de uma loja virtual, compuseram a mistura a ser avaliada.

As amostras de filé de tilápia (O.niloticus) foram cedidas por produtores da região de Palmeira das Missões/RS. As mesmas totalizaram aproximadamente 1 $\mathrm{kg}$ e foram transportadas congeladas $\left(-18^{\circ} \mathrm{C}\right)$ em embalagens plásticas transparentes e acondicionadas em caixa de polietileno, armazenadas sob congelamento, até o momento das análises.

O procedimento de preparação dos extratos foi conduzido de acordo com Bezerra (2012). Para os extratos de EM foi realizada uma pré-análise para verificar a melhor extração dos compostos, utilizando a mistura de etanol e água destilada $(20: 80, \mathrm{v} / \mathrm{v})$ com diferentes concentrações de EM, sendo selecionada a amostra com $30 \%$ (EM) para este trabalho. As extrações foram conduzidas em banho-maria por 30 minutos na temperatura de 40 으. Após, os macerados foram retirados do banho-maria, filtrados em algodão hidrófilo, acondicionados em tubos de $50 \mathrm{~mL}$ de capacidade e estocados sob refrigeração e ao abrigo da luz até o momento das análises. 0 procedimento de obtenção da associação dos três condimentos foi realizado a partir da mesma técnica de extração, porém foi utilizada a concentração de $10 \%$ (planta/solvente), a qual revelou a melhor extração de compostos.

A quantificação dos compostos fenólicos totais dos extratos de condimentos e EM foi determinada por espectrofotometria por meio da reação de oxirredução com o reagente de Folin-Ciocalteu usando uma curva de calibração com ácido gálico, e os valores são expressos em mg de equivalentes de ácido gálico (EAG) por g de extrato, conforme metodologia descrita por Singleton, Orthofer e Lamuela-Raventós (1999).

$O$ percentual de atividade antioxidante (\% AA) dos extratos de EM e de condimentos foi avaliado pela diminuição na quantidade do radical 2,2-difenil-1-picril-hidrazil (DPPH), conforme Brand-Williams, Cuvelier e Berset (1995). A capacidade de reduzir o DPPH é expressa em \% AA, sendo calculada em relação ao controle, segundo a expressão \% AA DPPH $=A C-(A A$ - AB) x 100/AC, em que AC é a absorbância do DPPH, $A A$ é a absorbância da amostra e $A B$ é a absorbância do branco de reação.

Depois da definição da concentração do extrato de EM e da associação dos condimentos, foram aplicados, em cada filé de tilápia descongelado, $10 \mathrm{~mL}$ de extrato de EM e $20 \mathrm{~mL}$ da mistura de condimentos (alecrim, orégano e tomilho).

Após, estas foram revestidas em papel alumínio, alocadas em sacos plásticos transparentes, congeladas por sete dias, e, posteriormente, aplicados os diferentes processamentos térmicos para a execução da análise de substâncias reativas ao ácido tiobarbitúrico (do inglês thiobarbituric acid reactive substances: TBARS).

Em relação aos tratamentos térmicos das amostras de pescado $(50 \mathrm{~g})$, após o descongelamento foram fritas (Ft) com $3 \mathrm{~mL}$ de óleo de soja em uma frigideira antiaderente ou assadas em forno elétrico (Ad).

Após serem submetidas aos diferentes modos de preparo, as amostras foram revestidas em papel alumínio, acondicionadas em sacos plásticos transparentes e congeladas por sete dias. $\mathrm{O}$ reaquecimento (Rq) ocorreu em forno micro-ondas por 20-30 segundos em potência máxima.

As análises foram realizadas com o filé de pescado in natura (IN), submetidos aos diferentes modos de preparo, designados como frito ( $\mathrm{Ft}$ ), assado (Ad) e reaquecido (Rq) e, ainda, com ou sem a adição do mix contendo a EM e os três condimentos.

A quantificação de produtos da peroxidação lipídica (TBARS) nas diferentes formas de preparo do filé de tilápia foi executada pela metodologia descrita por Buege e Aust (1978). As amostras foram preparadas a partir de $250 \mathrm{mg}$ de tecido muscular de pescado com KCl $150 \mathrm{mM}$ em homogeneizador, após fervura e centrifugação, formando um extrato (homogeneizado). Os valores foram expressos em nmol por $\mathrm{g}$ de amostra. 


\section{RESULTADOS E DISCUSSÃO}

Os resultados revelaram que, entre os condimentos analisados, o orégano foi o que apresentou o maior teor de compostos fenólicos totais, enquanto a associação de condimentos $10 \%$ com extrato de EM $30 \%$ apresentou o menor teor. Quanto ao percentual de inibição do radical DPPH (\%AA), o extrato de EM a $30 \%$, quando empregado isoladamente, mostrou o maior percentual de atividade antioxidante, e o alecrim, a menor atividade antioxidante ante o radical livre analisado (Tabela 1).

Tabela 1 - Compostos fenólicos totais e percentual de atividade antioxidante das amostras de condimentos, erva-mate (EM) e associação (mix) entre estes

\begin{tabular}{ccc}
\hline $\begin{array}{c}\text { Amostra } \\
\text { Condimentos }\end{array}$ & $\begin{array}{c}\text { Fenólicos totais } \\
\text { (mg EAG/g) }\end{array}$ & $\begin{array}{c}\text { Atividade } \\
\text { antioxidante (\%) }\end{array}$ \\
Orégano & 158,9 & 42,8 \\
Tomilho & 144,0 & 42,2 \\
Alecrim & 138,0 & 39,5 \\
EM 30\% & 151,8 & 59,4 \\
Mix condimentos & 133,2 & 42,9 \\
10\% + EM 30\% & & \\
\hline
\end{tabular}

EAG: equivalentes de ácido gálico.

Fonte: Dados da pesquisa/elaborada pelos autores.

Estudos recentes têm demonstrado o interesse por produtos naturais, preponderantemente de origem vegetal, com o objetivo de melhorar a conservação dos alimentos. Entre eles ameixa (JIANG; XIONG, 2016), pimenta, canela, gengibre, anis, romã, mirtilo, maçã (JIANG; XIONG, 2016), chá verde (NIKMARAM et al., 2018), orégano, uva (JIANG; XIONG, 2016; NIKMARAM et al., 2018; RIBEIRO et al., 2019), lichia, caqui (RIBEIRO et al., 2019), entre outros. A quantidade expressiva de compostos polifenólicos presentes nestes produtos é sugerida como a razão da atividade antioxidante, proporcionando efeitos benéficos à saúde de quem os consome tanto como alimento quanto como aditivo alimentício para restringir a oxidação (KANNER, 2007; BLUM-SILVA et al., 2015; SOUZA et al., 2015; JIANG; XIONG, 2016; BEZERRA et al., 2017; NIKMARAM et al., 2018).

Assim como registrado no atual estudo, o alto conteúdo de moléculas fenólicas na EM está bem documentado (BLUM-SILVA et al., 2015; COLPO et al., 2016; FERRARIO et al., 2018).

O processo de oxidação dos constituintes dos alimentos contribui desfavoravelmente para a sua preservação e diminui seu tempo de validade. $O$ intuito da inclusão de aditivos ou de algum processamen- to é prolongar o período no qual o alimento está apto para o consumo seguro, postergando sua deterioração (LAHMAR et al., 2018; POGORZELSKA et al., 2018).

Na Figura 1 estão apresentados os valores de TBARS das amostras de filé de tilápia in natura (IN) e submetidos aos diferentes tipos de processamentos (frito (Ft), assado ( $\mathrm{Ad})$ e reaquecido $(\mathrm{Rq})$ ) e com ou sem a inclusão do mix de condimentos e EM.

Figura 1 - TBARS nas amostras de filés de tilápia em diferentes formas de processamento térmico (Frito: $\mathrm{Ft}$, Assado: Ad, Reaquecido: Rq e IN: in natura) com e sem a adição do mix de condimentos e erva-mate (EM)

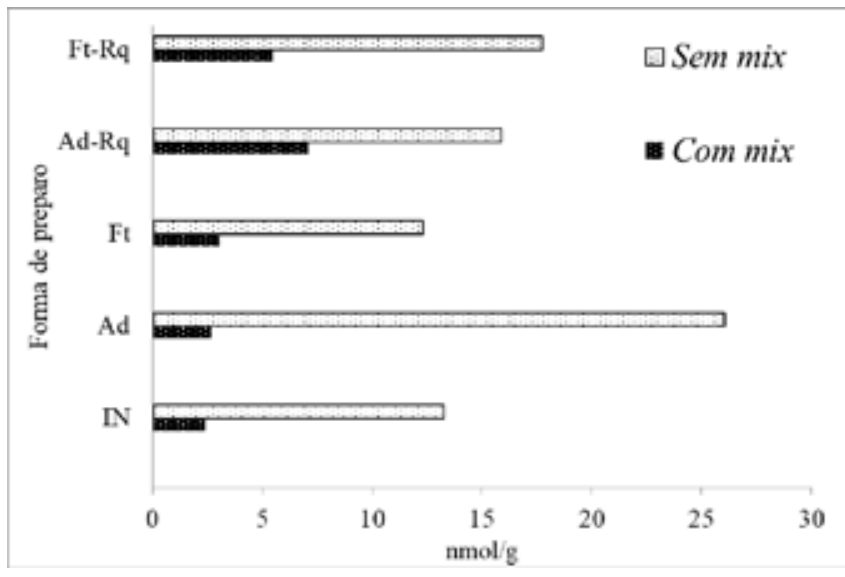

Fonte: Dados da pesquisa/elaborada pelos autores.

Os filés fritos e submetidos ao reaquecimento em micro-ondas ( $\mathrm{FtRq}$ ) exibiram um valor de TBARS aproximadamente três vezes maior do que aquele revelado pela amostra frita e reaquecida adicionada do mix. A forma de preparo assada e reaquecida (AdRq) apresentou o dobro da quantidade de TBARS do que aquela em que a amostra foi acondicionada com o mix.

Na condição frita, a adição do mix resultou em valores de TBARS em torno de $25 \%$ daquele apresentado pelo controle (Ft). A quantificação das TBARS do filé de tilápia assado sem mix (Ad) revelou que a adição da preparação de EM e condimentos diminui cerca de dez vezes o seu valor.

O filé in natura conservado com o mix apresentou uma redução de TBARS que atingiu cerca de $20 \%$ quando comparada àquela encontrada na preparação sem o mix (IN).

A condição de preparo do pescado, desde que associada ao $m i x$, não altera consideravelmente o nível de TBARS ( $A d=2,67 \mathrm{nmol} / \mathrm{g}$ e $\mathrm{Ft}=3,03 \mathrm{nmol} / \mathrm{g}$; $A d R q=7,07 \mathrm{nmol} / \mathrm{g}$ e $\mathrm{FtRq}=5,45 \mathrm{nmol} / \mathrm{g}$ ). O reaquecimento da preparação incluída do mix, entretanto, dobra a quantidade de TBARS presentes ( $\mathrm{Ad}=2,67$ $\mathrm{nmol} / \mathrm{g}$ e $\mathrm{AdRq}=7,07 \mathrm{nmol} / \mathrm{g} ; \mathrm{Ft}=3,03 \mathrm{nmol} / \mathrm{g}$ e FtRq $=5,45 \mathrm{nmol} / \mathrm{g}$ ). 
O nível elevado de lipídios presentes em produtos cárneos e afins é o principal determinante que impacta na sua degradação oxidativa (JIANG; XIONG, 2016; SMAOUl et al., 2016; LAHMAR et al., 2018; POGORZELSKA et al., 2018), fenômeno denominado rancificação, acarretando alteração de coloração, de sabor, de aroma e, consequentemente, prejudicando a aceitabilidade e encurtando o tempo hábil para o consumo (SMAOUl et al. 2016; POGORZELSKA et al., 2018). A proliferação de microrganismos imprime velocidade às vias metabólicas, gerando produtos enzimáticos finais que prejudicam a qualidade do produto (SMAOUI et al., 2016; LAHMAR et al., 2018).

A utilização de compostos naturais como aditivos alimentares tem sido requerida em detrimento de produtos químicos sintéticos (como BHT (butil-hidroxitolueno), BHA (butil-hidroxianisol) e TBHQ ( $t$-butil-hidroxiquinona)), com o intuito de diminuir ou anular os efeitos deletérios sobre a saúde consequentes do seu consumo (JIANG; XIONG, 2016; NIKMARAM et al., 2018; RIBEIRO et al., 2019). Os efeitos adversos destes agentes são o motivo pelo qual são determinados limites máximos para a sua ingestão, garantindo segurança ou consequências mínimas à saúde (RIBEIRO et al., 2019).

Os resultados do ensaio das TBARS mostraram a eficácia do mix de EM e condimentos na redução da rancidez oxidativa dos filés de tilápia submetidos aos diferentes processamentos térmicos aplicados, bem como do produto in natura (IN), uma vez que, sempre que foi usado, houve diminuição da oxidação lipídica.

A adição de I. paraguariensis tem se mostrado efetiva em melhorar a qualidade de produtos alimentícios tanto de origem vegetal quanto animal. Em suco misto de cenoura e laranja (FERRARIO et al., 2018), em ervilhas (YONNY et al., 2018) e em carne bovina (ZAWADZKI et al., 2017) foi demonstrada a melhora da estabilidade oxidativa. Quanto ao pescado, entretanto, os trabalhos são escassos e não consideram a EM como conservante para esta fonte alimentar proteica (HASSOUN; ÇOBAN, 2017; OZOGUL et al., 2017).

Fatores oxidantes, como xenobióticos, fármacos e alimentos oxidados, levam à geração de radicais livres e demais espécies reativas, estimulam processos inflamatórios e induzem à formação de toxinas (KANNER, 2007; MURUSSI et al., 2014; JIANG; XIONG, 2016), o que, indubitavelmente, acarreta, a longo prazo, o desenvolvimento de patologias/doenças e provoca impactos negativos à saúde dos seres humanos. O estresse oxidativo em proteínas e lipídios induz ao comprometimento funcional e fisiológico de moléculas enzimáticas, receptores, transportadores e de membranas e de organelas celulares (RIBEIRO et al., 2019). A exposição e/ou o consumo de compostos químicos antioxidantes mitigaria e/ou contribuiria para que tais consequências deletérias fossem minimizadas porque agem como scavengers de espécies reativas (BLUM-SILVA et al., 2015; COLPO et al., 2016; JIANG; XIONG, 2016). Neste contexto, já se tem confirmada suas eficácias como agentes antiproliferativos (WEBER, 2018), anti-inflamatório (SOUZA et al., 2015) e antimicrobiano (NIKMARAM et al., 2018).

\section{CONSIDERAÇÕES FINAIS}

A demonstração da eficiência do mix na redução da oxidação lipídica dos filés de tilápia, tanto sob as diferentes formas de preparo para o consumo quanto in natura, denota alternativas para a conservação do produto. Ademais, a revelação de que a associação entre a EM e o alecrim, o orégano e o tomilho diminuem a rancificação lipídica, mesmo após o reaquecimento do alimento previamente preparado para o consumo, prevê a possibilidade da utilização do mix como uma estratégia promissora na tecnologia de alimentos.

\section{REFERÊNCIAS}

BATTISTON, C. S. Z. et al. Caracterização físico-química e atividade antioxidante de chocolate branco com extrato de erva-mate. Revista Virtual de Química, v. 8, n. 6, p. 1.8781.888, 2016.

BEZERRA, A. S. Avaliação de compostos funcionais de grãos e extrato concentrado de cevada visando aplicações nutricionais. Santa Maria, RS, Brasil. 2012. Tese (Doutorado) Universidade Federal de Santa Maria, Programa de Pós-Graduação em Ciência e Tecnologia dos Alimentos, 2012.

BEZERRA, A. S. et al. Nutritional composition and antioxidant activity of non conventional food plants of the Southern region of Brazil. Brazilian Archives of Food, v. 2, n. 3, 182-188. 2017.

BLUM-SILVA, C. H. et al. The influence of leaf age on methylxanthines, total phenolic content, and free radical scavenging capacity of Ilex paraguariensis aqueous extract. Revista Brasileira de Farmacognosia, v. 25, p. 1-6, 2015.

BRAND-WILIAMS, W.; CUVELIER, M. E.; BERSET, C. Use of a free radical method to evaluate antioxidant activity. Food Science and Technology, v. 28, p. 25-30, 1995.

BUEGE, J. A.; AUST, S. D. Microsomal lipid peroxidation. Methods in Enzymology, v. 52, p. 302-309, 1978.

COLPO, A. C. et al. Yerba mate (Ilex paraguariensis St. Hill.) based beverages: How successive extraction influences the extract composition and its capacity to chelate iron and scavenge free radicals. Food Chemistry, v. 209, p. 185-195, 2016. 
EVANGELISTA-BARRETO, N. S. et al. Condições higiênicos sanitárias e grau de frescor do pescado comercializado no mercado de peixe em Cachoeira, Bahia. Brazilian Journal of Hygiene and Animal Sanity, 11, n. 1, p. 60-74, 2017.

FERRARIO, M. et al. Development and quality assessment of a turbid carrot-orange juice blend processed by UV-C light assisted by mild heat and addition of Yerba Mate (Ilex paraguariensis) extract. Food Chemistry, v. 269, p. 567-576, 2018.

HASSOUN, A.; ÇOBAN, Ö. E. Essential oils for antimicrobial and antioxidante applications in fish and other seafood products. Trends in Food Science \& Technology, v. 68, p. 2636, 2017.

HORN, R. C. et al. Avaliação dos níveis de estresse oxidativo em pacientes fumantes crônicos. Revista Contexto \& Saúde, v. 15, n. 29, p. 97-103, 2015.

IBGE. Instituto Brasileiro de Geografia e Estatística. Produção da pecuária municipal. 2017. Disponível em: https:// sidra.ibge.gov.br/tabela/3940\#resultado. Acesso em: 3 mar. 2019.

JIANG, J.; XIONG, Y. L. Natural antioxidants as food and feed additives to promote health benefits and quality of meat products: a review. Meat Science, v. 120, p. 107-117, 2016.

KANGARI, P. et al. Enzymatic antioxidant and lipid peroxidation evaluation in the newly diagnosed breast cancer patients in Iran. Asian Pacific Journal of Cancer Prevention, v. 19, n. 12, p. 3.511-3.515, 2018.

KANNER J. Dietary advanced lipid oxidation end products are risk factors to human health. Molecular Nutrition \& Food Research, v. 51, p. 1.094-1.101, 2007.

LAHMAR, A. et al. Prolonging shelf life of lamb cutlets packed under high-oxygen modified atmosphere by spraying essential oils from North-African plants. Meat Science, v. 39, p. 56-64, 2018.

MENDES, G. M.; RODRIGUES-DAS-DORES, R. G.; CAMPIDELI, L. C. Avaliação do teor de antioxidantes, flavonoides e compostos fenólicos em preparações condimentares. Revista Brasileira de Plantas Medicinais, v. 17, n. 2, p. 297-304, 2015.

MURUSSI, C. et al. Changes in oxidative markers, endogenous antioxidants and activity of the enzyme acetylcholinesterase in farmers exposed to agricultural pesticides - a pilot study. Ciência Rural, v. 44, n. 7, p. 1.186-1.193, 2014.

NIKMARAM, N. et al. Application of plant extracts to improve the shelf-life, nutritional and health-related properties of ready-to-eat meat products. Meat Science, v. 145, p. 245-255, 2018.

OZOGUL, Y. et al. Evaluation of effects of nanoemulsion based on herb essential oils (rosemary, laurel, thyme and sage) on sensory, chemical and microbiological quality of rainbow trout (Oncorhynchus mykiss) fillets during ice storage. LWT - Food Science and Technology, v. 75, p. 677-684, 2017.
POGORZELSKA, E. et al. Antioxidant potential of Haematococcus pluvialis extract rich in astaxanthin on colour and oxidative stability of raw ground pork meat during refrigerated storage. Meat Science, v. 135, p. 54-61, 2018.

RIBEIRO, J. S. et al. Natural antioxidants used in meat products: a brief review. Meat Science, v. 148, p. 181-188, 2019.

SINGLETON, V. L.; ORTHOFER, R.; LAMUELA-RAVENTÓS, R. $M$. Analysis of total phenols and other oxidation substrates and antioxidants by means of Folin-Ciocalteu reagent. Methods in Enzymology, v. 299, p. 152-178, 1999.

SMAOUI, S. et al. Bio-preservative effect of the essential oil of the endemic Mentha piperita used alone and in combination with BacTN635 in stored minced beef meat. Meat Science, v. 117, p. 196-204, 2016.

SOUZA, A. H. P. et al. Phytochemicals and bioactive properties of Ilex paraguariensis: an in-vitro comparative study between the whole plant, leaves and stems. Food Research International, v. 78, p. 286-294, 2015.

VISIOLI, F. Rusting the pipes: Ingestion of oxidized lipids and vascular disease. Vascular Pharmacology, v. 62, n. 2.014, p. 47-48, 2014.

WANG, M.; LU, M. Tilapia polyculture: a global review. Aquaculture Research, v. 47, p. 2.363-2.374, 2016.

WEBER, A. V. Potencial efeito antioxidante e antiproliferativo do extrato aquoso de Carya illinoinensis sobre células tumorais. Revista Contexto \& Saúde, v. 18, n. 35, p. 91-94, 2018.

YONNY, M. E. et al. Enhancement in the oxidative stability of green peas by llex paraguariensis addition in a blanching process before their refrigerated and frozen storage. $L W T-$ Food Science and Technology, v. 91, p. 315-321, 2018.

ZAWADZKI, A. et al. Mate extract as feed additive for improvement of beef quality. Food Research International, v. 99, p. 336-347, 2017. 